\title{
Evolution and variation of the nifD and hupL elements in the heterocystous cyanobacteria
}

\author{
Correspondence \\ Susan R. Barnum \\ barnumsr@muohio.edu
}

\author{
Brian J. Henson, ${ }^{1}$ Linda Hartman, ${ }^{2}$ Linda E. Watson ${ }^{3}$ \\ and Susan R. Barnum² \\ ${ }^{1}$ Department of Pediatrics, Children's Hospital of Pittsburgh of UPMC, USA \\ ${ }^{2}$ Department of Botany, Miami University, USA \\ ${ }^{3}$ Department of Botany, Oklahoma State University, USA
}

\section{INTRODUCTION}

The heterocystous cyanobacteria are unique in that they develop heterocysts, which are specialized cells with thickened cell walls that prevent nitrogenase from coming into contact with oxygen, thereby spatially separating photosynthesis from nitrogen fixation (Adams \& Duggan, 1999; Böhme, 1998; Wolk et al., 1994). Heterocysts supply adjacent vegetative cells with reduced nitrogen and, in return, the vegetative cells supply the heterocysts with photosynthates (Adams \& Duggan, 1999; Böhme, 1998; Wolk et al., 1994). Heterocyst differentiation is a complex process involving up to 1000 different genes, or approximately $15-25 \%$ of the cyanobacterial genome (Adams \& Duggan, 1999; Lynn et al., 1986). Several developmentally regulated DNA rearrangements accompany heterocyst differentiation, which involves the removal of a segment of DNA that coincides with a developmental shift or differentiation process. Cellular differentiation and developmentally regulated DNA rearrangements are uncommon in prokaryotes, with the best studied examples being sporulation in Bacillus subtilis and

Abbreviations: AIC, Akaike information criterion; hLRTs, hierarchical likelihood ratio test; ML, maximum-likelihood; ORF, open reading frame.

The GenBank/EMBL/DDBJ accession numbers for the gene sequences determined in this study are given in Table 3 .

Two supplementary figures are available with the online version of this paper.
Clostridium difficile, as well as heterocyst differentiation in cyanobacteria (Kunkel et al., 1990; Haselkorn, 1992; Haraldsen \& Sonenshein, 2003).

In cyanobacteria, DNA rearrangements occur within the nifD, $f d x N$ and $h u p L$ genes, which encode a subunit of nitrogenase, the ferredoxin protein and a subunit of the membrane-bound uptake hydrogenase, respectively (Golden et al., 1985, 1987, 1991; Mulligan et al., 1988; Oxelfelt et al., 1985; Haselkorn, 1992; Carrasco \& Golden, 1995; Carrasco et al., 1995; Wolk et al., 1994). In each of these three genes, specific segments of DNA, hereafter referred to as DNA elements, are independently excised from the genome during the later stages of heterocyst differentiation by site-specific recombination between direct repeats that flank each element (Adams \& Duggan, 1999; Haselkorn, 1992; Carrasco et al., 1995). The length and nucleotide sequence of the direct repeats varies for each element type (Adams \& Duggan, 1999) and each element encodes its own site-specific recombinase responsible for its excision (Adams \& Duggan, 1999; Böhme, 1998). All three DNA elements have been found in Nostoc sp. strain PCC 7120 (Golden et al., 1985, 1987, 1991), but otherwise little is known about their co-occurrence and distribution within the heterocystous cyanobacteria.

The three types of DNA elements vary in their size and pattern of excision. An $11 \mathrm{~kb}$ element is present within the nifD gene of Nostoc sp. strain PCC 7120. The NifD gene is 
part of the nifHDK operon, which encodes nitrogenase (Mazur \& Chui, 1982). Expression of sequences downstream of the element in the nifHDK operon and nitrogen fixation are contingent on the removal of this element. It is excised between $11 \mathrm{bp}$ direct repeats (CGGAGTAATCC) that flank the element by XisA, which is encoded within the element (Brusca et al., 1989, 1990; Golden et al., 1985, 1987, 1991; Lammers et al., 1986, 1990; Haselkorn, 1992). Many other potential open reading frames (ORFs) are located within the element; although it is not believed that any gene other than xisA is required for excision (Lammers et al., 1986, 1990; Brusca et al., 1990). A $55 \mathrm{~kb}$ element is embedded within the $f d x N$ gene of Nostoc sp. strain PCC 7120. The $f d x N$ gene is part of the nifBSU, $f d x N$ operon (Golden et al., 1987, 1988; Mulligan et al., 1988; Carrasco et al., 1994) and removal of the element is required for expression of sequences downstream of the element. The element is flanked by 5 bp direct repeats (TATTC) and it is excised by XisF and its accessory proteins $\mathrm{XisH}$ and XisI, all of which are encoded within the $f d x N$ element (Ramaswamy et al., 1997). Embedded within the hupL gene of Nostoc sp. strain PCC 7120 is a $9.4 \mathrm{~kb}$ element (Carrasco et al., 1995, 2005). This element is flanked by 16 bp direct repeats (CCATATAACTGCTGTG) and it is excised by the recombinase $\mathrm{XisC}$, which is encoded within the element (Carrasco et al., 1995, 2005) (Fig. 2). It is believed that no other functional products are encoded within the hupL element (Carrasco et al., 1995, 2005).

Although these programmed DNA rearrangements have received moderate attention in the literature, many questions remain regarding their evolution, variation and regulation of excision. It is not known how widespread these elements are in heterocystous cyanobacteria, however the nifD and hupL elements have been found in a number of strains (Tables 1 and 2, respectively), whereas the $f d x N$ element has only been found in three strains: Nostoc sp. strain Mac, Anabaena sp. strain PCC 7122 and Anabaena sp. strain M131 (Carrasco \& Golden, 1995). It is generally believed that only heterocystous cyanobacteria of Subsection IV harbour the elements (Saville et al., 1987; Kallas et al., 1985; Lammers et al., 1990); however, a recent study reported the presence of the hupL element (or a portion of xisC) in Fischerella sp. strain PCC 7521 (Subsection V) and Leptolyngbya sp. strain PCC 73110 (a nonheterocystous Subsection III strain) (Table 2) (Tamagnini et al., 2000). Within Subsection IV, the distribution of the nifD and hupL elements appears to be haphazard and their pattern of occurrence does not appear to be linked (Happe et al., 2000; Meeks et al., 2001).

The origins of these DNA elements remain unknown. It has been suggested that the nifD, hupL and $f d x N$ elements are the remnants of ancient viral infections and have lost the ability to self-replicate (Haselkorn, 1992; Henson et al., 2005). The $f d x N$ element has sequence similarity to the Bacillus subtilis SKIN element $(48 \mathrm{~kb})$ that is excised from within the sigK gene during sporulation by the SpoIVCA recombinase (Kunkel et al., 1990; Haselkorn, 1992;
Carrasco et al., 1994). Both XisF and SpoIVCA are members of the resolvase/invertase or serine family of recombinases (Smith \& Thorpe, 2002). XisA and XisC belong to the tyrosine or phage integrase family of sitespecific recombinases (Nunes-Düby et al., 1998). The tyrosine integrases are characterized by a conserved $\mathrm{R}-\mathrm{H}-$ $\mathrm{R}-\mathrm{Y}$ tetrad within the active site (Voziyanov et al., 1999; Nunes-Düby et al., 1998); however, XisA and XisC have an altered active site with a tyrosine as opposed to a histidine (R-Y-R-Y) (Nunes-Düby et al., 1998). XisA and XisC are $61 \%$ similar at the nucleotide level and $43 \%$ similar at the amino acid level (Carrasco et al., 1995). BLAST (basic local alignment search tool) searches indicate that they are more similar to each other than to any other sequence in GenBank. The similarities between xis $A$ and $x i s C$ suggest that they may share a common ancestor and this also suggests that the nifD and hupL elements themselves may share a common evolutionary origin and may have arisen by subsequent duplication and divergence prior to their insertion within the cyanobacterial lineage.

Our objectives were to examine the structural and compositional variation within the nifD and hupL elements and examine whether or not they are of viral origin. This was accomplished by comparative analysis of full-length nifD and hupL gene sequences and phylogenetic analysis of xisA and $x i s C$ gene sequences.

\section{METHODS}

Cyanobacterial strains (Table 3) were obtained from the Pasteur Culture Collection (PCC) and American type Culture Collection (ATCC) and were grown in an illuminated shaking incubator at $27{ }^{\circ} \mathrm{C}$ in a variation of BG-11 media [BG- $\left.11_{0}+\mathrm{NaHCO}_{3}(5 \mathrm{mM})\right]$, which contained reduced nitrate. Genomic DNA was extracted using a Puregene DNA Isolation kit (Gentra Systems) with a slight modification (Henson et al., 2002).

Amplification of full-length nifD and hupL elements was accomplished with the Expand Long Template PCR system (Roche). For the nifD element, the primers used for PCR amplification were $5^{\prime}$ TTTCCGCCAAATGCACTCTTG-3' and $5^{\prime}$-GCAAACCGTCGTAACCGTGAT-3', which are located within the flanking regions of the nifD direct repeats. To amplify the hupL element, the primers used for PCR amplification were 5'-GGCGCACATCACACCACCAGGC-3' and 5' GCCCTGTTTGGCGGACAATGGC-3', which are located within the flanking regions of the hupL direct repeats. PCR conditions for amplifying full-length elements were as follows: an initial hold at $94{ }^{\circ} \mathrm{C}$ for $2 \mathrm{~min}, 10$ cycles of denaturation at $94{ }^{\circ} \mathrm{C}$ for $10 \mathrm{~s}$, annealing at $55{ }^{\circ} \mathrm{C}$ for $30 \mathrm{~s}$, and extension at $68{ }^{\circ} \mathrm{C}$ for $10 \mathrm{~min}$ followed by 15 cycles of denaturation at $94{ }^{\circ} \mathrm{C}$ for $10 \mathrm{~s}$, annealing at $55{ }^{\circ} \mathrm{C}$ for $30 \mathrm{~s}$, extension at $68{ }^{\circ} \mathrm{C}$ for $10 \mathrm{~min}$, with each cycle increasing $20 \mathrm{~s}$ in duration, and a final extension of $68{ }^{\circ} \mathrm{C}$ for $7 \mathrm{~min}$. PCR products were verified by gel electrophoresis on $0.8 \%$ agarose and purified with the QIAquick Gel Extraction kit (Qiagen). The cyanobacterial strains from which nifD and hupL elements were sequenced are listed in Tables 1 and 2, respectively.

Amplification of partial $x i s A$ and $x i s C$ genes was accomplished with Taq DNA polymerase (New England Biolabs). The cyanobacterial strains from which partial $x i s A$ and $x i s C$ sequences were generated are listed in Tables 1 and 2, respectively. Primers used to amplify xisA were $5^{\prime}$-CTGCGTTTGAAGTCTGCAAAGAC-3' and 5'-CGTAAAC- 
Table 1. Cyanobacteria examined for the presence of the nifD element

Strains are separated by taxonomic Subsection. Presence refers to whether or not the element has been detected. Method refers to whether the element was detected by Southern hybridization (S) or by DNA sequencing (D). Y, Yes; N, no; NA, not available; C, completely sequenced.

\begin{tabular}{|c|c|c|c|c|}
\hline Strain & Presence & Method & Length sequenced & Reference \\
\hline \multicolumn{5}{|l|}{ Subsection I } \\
\hline Aphanocaps sp. ATCC 27178 & $\mathrm{~N}$ & $\mathrm{~S}$ & NA & Saville et al. (1987) \\
\hline Anacystis nidulans $\mathrm{R} 2$ & $\mathrm{~N}$ & $S$ & NA & Saville et al. (1987) \\
\hline Coccochloris peniocystis & $\mathrm{N}$ & $S$ & NA & Saville et al. (1987) \\
\hline Cyanothece sp. PCC 7424 & $\mathrm{~N}$ & $S$ & NA & Kallas et al. (1985) \\
\hline Gloeocapsa sp. ATCC 27191 & $\mathrm{~N}$ & $S$ & NA & Saville et al. (1987) \\
\hline Synechococcus cedrorum & $\mathrm{N}$ & $S$ & NA & Saville et al. (1987) \\
\hline Synechococcus sp. PCC 6301 & $\mathrm{~N}$ & S & NA & Kallas et al. (1985) \\
\hline Synechococcus sp. PCC 7335 & $\mathrm{~N}$ & S & NA & Kallas et al. (1985) \\
\hline Synechococcus sp. PCC 7425 & $\mathrm{~N}$ & S & NA & Kallas et al. (1985) \\
\hline Synechocystis sp. ATCC 29109 & $\mathrm{~N}$ & $S$ & NA & Saville et al. (1987) \\
\hline \multicolumn{5}{|l|}{ Subsection III } \\
\hline Leptolyngbya sp. ATCC 29126 & $\mathrm{~N}$ & $S$ & NA & Saville et al. (1987) \\
\hline Leptolyngbya sp. PCC 73110 & $\mathrm{~N}$ & $S$ & NA & Kallas et al. (1985) \\
\hline Pseudoanabaena sp. ATCC 29210 & $\mathrm{~N}$ & S & NA & Saville et al. (1987) \\
\hline Pseudoanabaena sp. PCC 7409 & $\mathrm{~N}$ & $S$ & NA & Kallas et al. (1985) \\
\hline \multicolumn{5}{|l|}{ Subsection V } \\
\hline Fischerella sp. ATCC 27929 & $\mathrm{~N}$ & $S$ & NA & Saville et al. (1987) \\
\hline Mastigocladus sp. ARM 351 & $\mathrm{~N}$ & $S$ & NA & Prasanna \& Kaushik (1995) \\
\hline \multicolumn{5}{|l|}{ Subsection IV } \\
\hline Anabaena azollae & $\mathrm{N}$ & $\mathrm{S}$ & NA & Meeks et al. (1988) \\
\hline Anabaena sp. ATCC 33047 & $\mathrm{Y}$ & $\mathrm{D}$ & $\mathrm{C}$ & Henson et al. (2005) \\
\hline Anabaena sp. PCC 7108 & $\mathrm{Y}$ & $\mathrm{D}$ & xisA (700 bp) & This study \\
\hline Anabaena. variabilis & $\mathrm{Y}$ & $\mathrm{D}$ & $\mathrm{C}$ & Brusca et al. (1989) \\
\hline Calothrix sp. PCC 7601 & $\mathrm{Y}$ & $\mathrm{S}$ & NA & Kallas et al. (1985) \\
\hline Cylindrospermum sp. PCC 7604 & $\mathrm{Y}$ & $\mathrm{D}$ & $x i s A(500 \mathrm{bp})$ & This study \\
\hline Cylindrospermum sp. PCC 7417 & $\mathrm{Y}$ & $\mathrm{D}$ & $\mathrm{C}$ & This study \\
\hline Nostoc sp. PCC 7120 & $\mathrm{Y}$ & $\mathrm{D}$ & $\mathrm{C}$ & Kaneko et al. (2001) \\
\hline Nostoc sp. PCC 7121 & $\mathrm{Y}$ & $\mathrm{D}$ & $x i s A(700 \mathrm{bp})$ & This study \\
\hline Nostoc sp. PCC 7906 & $\mathrm{Y}$ & $\mathrm{D}$ & $x i s A(500 \mathrm{bp})$ & This study \\
\hline Tolypothrix sp. PCC 7415 & $\mathrm{Y}$ & $\mathrm{D}$ & $x i s A(700 \mathrm{bp})$ & This study \\
\hline Nostoc sp. PCC 6314 & $\mathrm{Y}$ & $\mathrm{D}$ & xisA (700 bp) & This study \\
\hline Nostoc sp. PCC 7118 & $\mathrm{Y}$ & $\mathrm{D}$ & xisA (700 bp) & This study \\
\hline Nostoc sp. PCC 7416 & $\mathrm{Y}$ & $\mathrm{D}$ & $x i s A(700 \mathrm{bp})$ & This study \\
\hline Nostoc sp. PCC 7524 & $\mathrm{Y}$ & $\mathrm{D}$ & $x i s A(700 \mathrm{bp})$ & This study \\
\hline Nostoc punctiforme & $\mathrm{Y}$ & $\mathrm{D}$ & $\mathrm{C}$ & Meeks et al. (2001) \\
\hline Nostoc sp. strain Mac & $\mathrm{Y}$ & $\mathrm{S}$ & NA & Meeks et al. (1994) \\
\hline Scytonema sp. PCC 7110 & $\mathrm{Y}$ & $\mathrm{D}$ & xisA (700 bp) & This study \\
\hline Scytonema sp. ARM 428 & $\mathrm{Y}$ & S & none & Prasanna \& Kaushik (1995) \\
\hline Scytonematopsis sp. ARM 428 & $\mathrm{~N}$ & $S$ & NA & Prasanna \& Kaushik (1995) \\
\hline Tolypothrix ceylonica ARM397 & $\mathrm{Y}$ & S & none & Prasanna \& Kaushik (1995) \\
\hline
\end{tabular}

CAAAAACTGCTAACATCC-3'. To amplify $x i s C$, the primers $5^{\prime}$ GCAGACTCCAACCGACTGGTTCGC-3' and 5'-TATGATTTGTCTCTAGGGATACCA-3' $3^{\prime}$ were used. PCR conditions included: an initial hold at $94{ }^{\circ} \mathrm{C}$ for $2 \mathrm{~min}, 35$ cycles of denaturation at $94{ }^{\circ} \mathrm{C}$ for $20 \mathrm{~s}$, annealing at $55{ }^{\circ} \mathrm{C}$ for $15 \mathrm{~s}$, and extension at $72{ }^{\circ} \mathrm{C}$ for $1 \mathrm{~min}$. PCR products were verified by gel electrophoresis on $0.8 \%$ agarose and were purified with a QIAquick Gel Extraction kit (Qiagen).

Sequencing of purified PCR products was accomplished with a DYEnamic ET Terminator cycle sequencing kit (Amersham Biosciences) using capillary electrophoresis on ABI 310, ABI 3100 and ABI 3730 Genetic Analyzers (Applied Biosystems). Both the forward and reverse DNA strands of the PCR products (full-length
nifD and hupL elements and partial xis $A$ and $x i s C$ genes) were sequenced to ensure that the nucleotide sequences were accurate and that no artefacts of PCR were present. Sequences were deposited in GenBank and the accession numbers are listed in Table 3.

BLAST searches were carried out using complete nifD and hupL element sequences, as well as smaller portions of each element (ranging from $\sim 200$ to $1500 \mathrm{bp}$ ) to determine regions of similarity with other sequences in the database. The BLAST programs used were: quickly search for highly similar sequences (MEGABLAST), quickly search for divergent sequences (discontiguous MEGABLAST), nucleotide-nucleotide BLAST (BLASTN), search for short, nearly exact matches and translated query vs protein database (BLASTX). Databases that 
Table 2. Cyanobacterial strains that have been examined for the presence of the hupL element

Strains are separated by Subsection. Presence refers to whether or not the element has been detected. Method refers to whether the element was detected by Southern hybridization (S) or by DNA sequencing (D). Y, Yes; N, no; NA, not available; C, completely sequenced.

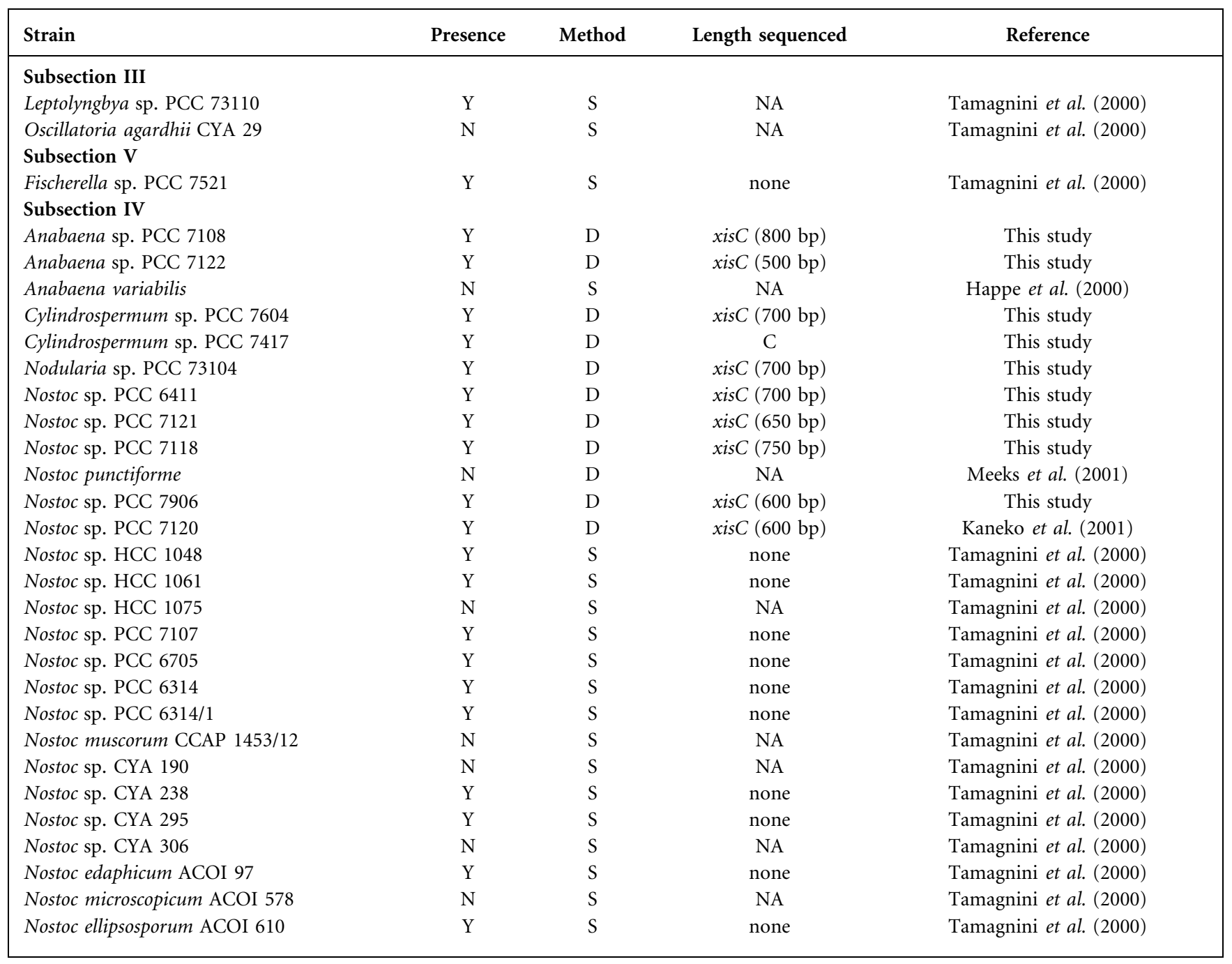

were searched included NR, EST, WGS and individual genomes. All elements were searched against completely sequenced microbial genomes, including eubacteria and archaea.

Phylogenetic analyses of $x i s A$ and $x i s C$ nucleic acid sequences were accomplished with PAUP ${ }^{*} 4.0 \mathrm{~b} 10$ using parsimony, distance and maximum-likelihood (ML) criteria. The xisA tree was rooted with $x i s C$ from Nostoc sp. strain PCC 7120 and the $x i s C$ tree was rooted with the xisA from Nostoc sp. strain PCC 7120. To construct the data matrices, inferred amino acid sequences were aligned using CLUSTAL $\mathrm{W}$ (Thompson et al., 1994) with gaps inserted for optimal alignment. The amino acid alignments were manually adjusted using MacClade 4.0. Nucleotide alignments were generated using Codon Align 2.0 (Hall, 2004), which constructs the nucleotide alignment based on the amino acid alignment, with gaps inserted between codons rather than within them.

Prior to performing ML analyses, Modeltest 3.7 was used to determine the evolutionary model that best fitted the $x i s A$ and $x i s C$ data (Posada \& Crandall, 1998). Modeltest uses the hierarchical likelihood ratio test (hLRTs) and the Akaike information criterion (AIC) for selecting the best fit model. For xisA, Modeltest selected $\operatorname{TrN}+\mathrm{G}(-\ln \mathrm{L}=$ 7176.2539) for the hLRTs and GTR $+\mathrm{G}(-\operatorname{lnL}=7145.8521)$ for AIC. For $x i s C$, Modeltest selected $\mathrm{HKY}+\mathrm{G}(-\ln \mathrm{L}=4562.8667)$ for the hLRTs and GTR $+\mathrm{G}(-\operatorname{lnL}=4544.7305)$ for the AIC.

ML analyses were conducted using the heuristic search option, with the likelihood settings corresponding to each of the models selected by Modeltest. The parameters were as follows: no molecular clock was enforced, starting branch lengths were determined via the RogersSwofford approximation method, trees with likelihoods that were $5 \%$ or further from the target score were rejected, branch-length optimization equalled one-dimensional Newton Raphson with pass where the limit $=20$ and $\Delta=1 \mathrm{e}-06$; starting trees were obtained via stepwise addition, sequence addition was random, one tree was held at each step during stepwise addition, the branch swapping algorithm tree bisection reconnection (TBR) was used, steepest descent option was not in effect, maxtrees was set to 50000 , branches were collapsed if the branch lengths were equal to or less than $1 \mathrm{e}-08$, the multrees option was in effect, and topological constraints were not enforced. Bootstrap values were calculated for 1000 replicates to evaluate branch support using parsimony criteria (Bremer, 1994; Felsenstein, 1985; Huelsenbeck et al., 1995). 
Table 3. Sequences used in this study and their GenBank accession numbers

NA, Not available.

\begin{tabular}{|c|c|c|}
\hline Strain & nifD element & hupL element \\
\hline Anabaena sp. ATCC 33047 & AY299473 & NA \\
\hline Anabaena sp. PCC 7108 & HQ284169 & HQ284178 \\
\hline Anabaena sp. PCC 7122 & NA & HQ284177 \\
\hline Anabaena variabilis & NC_007413* & NA \\
\hline $\begin{array}{l}\text { Cylindrospermum sp. PCC } \\
7417\end{array}$ & HQ284186 & HQ284186 \\
\hline $\begin{array}{l}\text { Cylindrospermum sp. PCC } \\
7604\end{array}$ & HQ284176 & HQ284184 \\
\hline Nodularia sp. PCC 73104 & NA & HQ284182 \\
\hline $\begin{array}{l}\text { Nodularia spumigena } \\
\text { CCY9414 }\end{array}$ & NZ_AAVW00000000* & NZ_AAVW00000000* \\
\hline Nostoc punctiforme & NC_010628* & NA \\
\hline Nostoc sp. PCC 6314 & HQ284167 & NA \\
\hline Nostoc sp. PCC 6411 & NA & HQ284179 \\
\hline Nostoc sp. PCC 7118 & HQ284170 & HQ284180 \\
\hline Nostoc sp. PCC 7120 & NC_003272* & NC_003272* \\
\hline Nostoc sp. PCC 7121 & HQ284174 & HQ284181 \\
\hline Nostoc sp. PCC 7416 & HQ284168 & NA \\
\hline Nostoc sp. PCC 7524 & HQ284172 & NA \\
\hline Nostoc sp. PCC 7906 & HQ284175 & HQ284184 \\
\hline Scytonema sp. PCC 7110 & HQ284173 & NA \\
\hline Tolypothrix PCC 7415 & HQ284171 & NA \\
\hline
\end{tabular}

${ }^{\star}$ Genome sequences retrieved from GenBank and not derived from this study.

\section{RESULTS}

\section{Structural variation of the nifD element}

To date, the nifD element has been completely sequenced from six cyanobacterial strains (Table 3 ), and there is considerable variation in size (ranging from 4 to $24 \mathrm{~kb}$ ) and sequence composition among them (Henson et al., 2005) (Fig. 1). With the exception of the nifD elements from Nostoc sp. strain PCC 7120 and Anabaena variabilis, which are very similar to each other, the elements from the other strains were unique. There were conserved regions within all nifD elements (Fig. 1). The $5^{\prime}$ and $3^{\prime}$ flanking regions were conserved (Fig. 1) and each element was flanked by $11 \mathrm{bp}$ direct repeats (CGGAGTAATCC). However, in Anabaena sp. strain ATCC 33047 (Henson et al., 2005) and Nostoc sp. strain PCC 7416, the direct repeat nearest xisA differed by one nucleotide each, CGGAGTAATTC in Anabaena sp. strain ATCC 33047 and CGGAGTAATCA in Nostoc sp. strain PCC 7416 (the variable nucleotide is in bold face and underscored). There were regions shared among individual elements that were not universally present (Fig. 1). For example, the hypothetical protein alr1449, originally designated ORF2 (Lammers et al., 1990; Henson et al., 2005) was conserved in all nifD elements, except in Nodularia spumigena CCY 9414 (Fig. 1). Others have suggested that alr1449 may be expressed in Nostoc sp. strain PCC 7120
(Rice et al., 1982). While some members of the serine family of site-specific recombinases require additional proteins for recombination, members of the tyrosine family, including $x i s A$ and $x i s C$, do not (Smith \& Thorpe, 2002; Nunes-Düby et al., 1998). This information, combined with its absence in Nodularia spumigena CCY 9414, suggests that alr1449 is not required for excision. The Nostoc sp. strain PCC 7120 and A. variabilis nifD elements were $\sim 70 \%$ identical over their entire length, with the only difference being the absence of alr1443, asr1451, asr1452 and asr1453 in A. variabilis (Fig. 1). The element in Nostoc punctiforme contained a portion of all1445, which it shared with Nostoc sp. strain PCC 7120 and A. variabilis (Fig. 1). The nifD elements from Cylindrospermum sp. strain PCC 7417 and Nostoc punctiforme both contained the tRNA-Asn and ctiA genes. The tRNA-Asn gene encodes the tRNA for asparagine. The function of the ctiA gene remains unknown, but it is similar to the family of membranebound stomatin/prohibitin genes, which are found throughout the eukaryotes and the prokaryotes. The tRNA-Asn and ctiA region was conserved in A. variabilis, Nostoc sp. strain PCC 7120, Nostoc punctiforme and Cylindrospermum sp. strain PCC 7417; although, it was only located within the nifD element of the latter two.

BLAST searches of the sequenced nifD elements confirmed the expected similarities to the conserved regions of other 


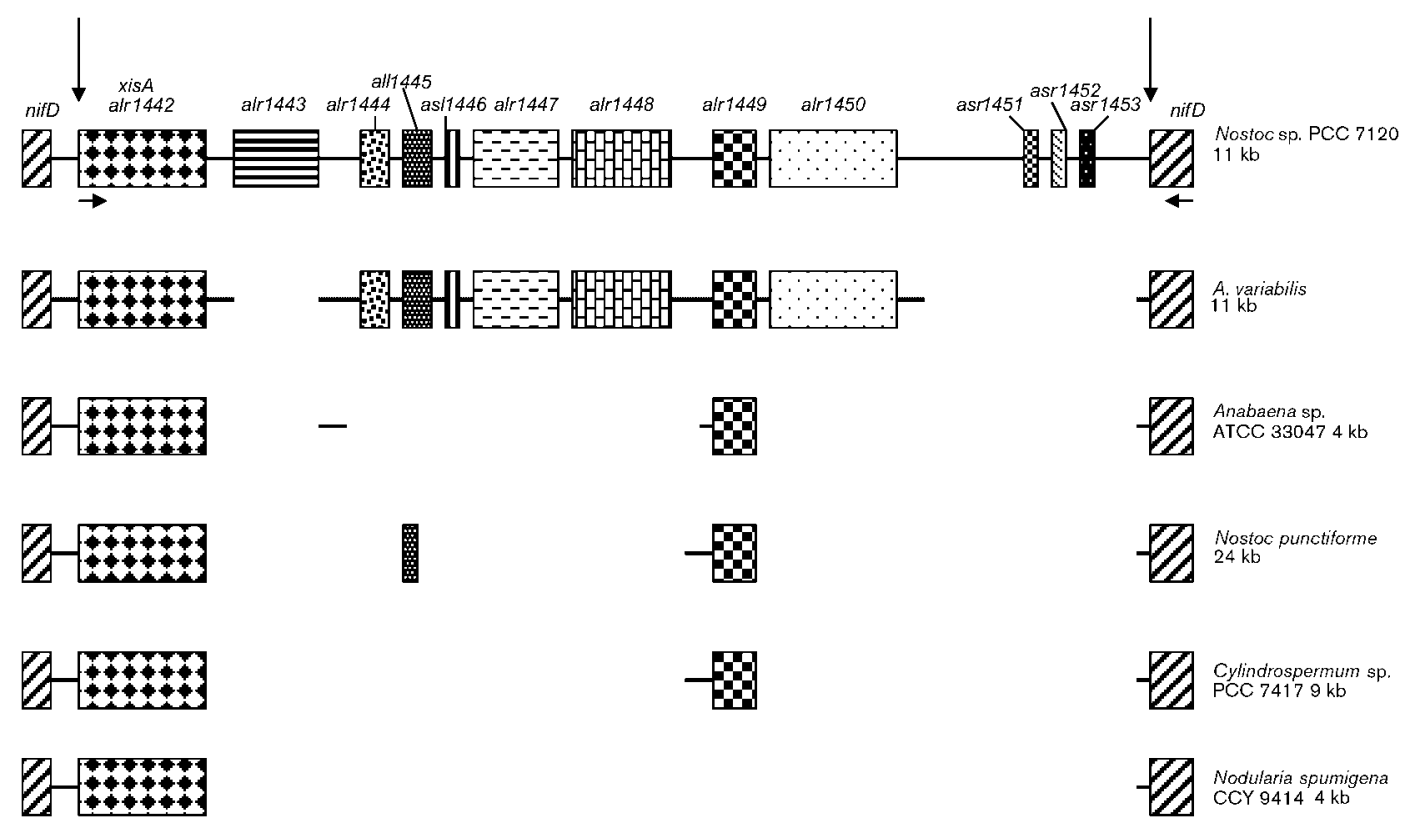

Fig. 1. A diagram comparing the similarity between nifD elements. All ORFs of the Nostoc sp. strain PCC 7120 nifD element (top) are represented by labelled boxes with different graphical patterns. The direct repeats are represented by vertical arrows and the horizontal arrows indicate the direction of transcription for nifD and xis $A$. Regions of the $A$. variabilis, Anabaena sp. strain ATCC 33047, Nostoc punctiforme, Cylindrospermum sp. strain PCC 7417 and Nodularia spumigena CCY 9414 nifD elements that are similar to Nostoc sp. strain PCC 7120 genes are represented by boxes with identical graphical patterns and intergenic regions are represented by horizontal bars.

nifD elements (Fig. 1). They also revealed that particular regions of these elements were similar to a variety of other sequences not found in other nifD elements. For instance the hypothetical gene alr1444 (present in the nifD elements of Nostoc sp. strain PCC 7120 and A. variabilis) was similar to a number of cyanobacterial genes including $p e b A B$, which is involved in the synthesis of the photosynthetic pigment phycoerythrobilin (Alvey et al., 2003), sll1232 in Synechocystis sp. strain PCC 6803, and glr0577 and gll1896 in Gloeobacter sp. strain PCC 7421. The asl1446 gene, present in the nifD elements of Nostoc sp. strain PCC 7120 and A. variabilis, was similar to putative proteins within a number of cyanobacteria and, in some cases, to multiple genes within individual genomes. In addition, a region of the nifD element in $A$. variabilis was similar to $r b p G$, which encodes an RNA-binding protein that is located in multiple places within the A. variabilis genome (Maruyama et al., 1999).

In addition to the tRNA-Asn and ctiA genes mentioned earlier, the $24 \mathrm{~kb}$ nifD element in Nostoc punctiforme contained regions that were similar to eubacterial kinases, the Synechocystis sp. strain PCC 6803 slr1963 gene that is believed to encode solanesyl diphosphate synthase involved in the formation of isoprenoid quinones (Okada et al., 1997), and the Nostoc sp. strain PCC 7120 alr1077 gene, which encodes a probable carboxymethylenebutenolidase (Sazuka, 2003).
The Nodularia spumigena CCY 9414 nifD element contained a 200 bp region which was similar to the Nostoc sp. strain PCC $7524 N s p V$ endonuclease. BLAST searches of the nifD element of Anabaena sp. strain ATCC 33047 revealed a $125 \mathrm{bp}$ region of this element, composed mostly of repeating CATCTT units, which was similar to several viruses (Rabbit fibroma virus and Rat cytomegalovirus) (Henson et al., 2005). Other than the conserved regions found in the other sequenced nifD elements, the $9 \mathrm{~kb}$ nifD element from Cylindrospermum sp. strain PCC 7417 had little similarity to any other known sequence.

\section{Structural variation of the hupL element}

To date, the hupL element from three cyanobacterial strains has been sequenced and there was variation in size (ranging from 7 to $9.4 \mathrm{~kb}$ ) and composition among them. Most of each element was unique; but there were also conserved regions (Fig. 2). The $5^{\prime}$ and $3^{\prime}$ flanking regions, including $x i s C$, were conserved. The hupL element was flanked by 16 bp direct repeats, CCATATAACTGCTGTG. However, in the hupL elements of Cylindrospermum sp. strain PCC 7417 and Nodularia spumigena CCY 9414, the direct repeat nearest $x i s C$ differed by one nucleotide, CCATATAACTGCTATG (variable nucleotide in bold and underscored). The region encompassing asr0680, alr0681 and asr0682 was conserved in all hupL elements, however the 
arrangement of these hypothetical genes was different in Cylindrospermum sp. strain PCC 7417 compared with the other two strains. In Nostoc sp. strain PCC 7120 and Nodularia spumigena CCY 9414, they were encoded as asr0680, alr0681 and asr0682 (5' to $3^{\prime}$ ), whereas in Cylindrospermum sp. strain PCC 7417 they were encoded in the order: asr0682, asr0680 and alr0681 (Fig. 2). Although it cannot be resolved with absolute certainty, we hypothesize that asr0682 (as it appears in Cylindrospermum sp. strain PCC 7417) is the result of a translocation. This is supported by comparative sequence analysis of the three sequenced hupL elements. The intergenic region between asr0681 and asr0682 (present in Nodularia spumigena CCY 9414 and Nostoc sp. strain PCC 7120) was absent in Cylindrospermum sp. strain PCC 7417. In Cylindrospermum sp. strain PCC 7417, the end of asr0682 was located exactly $92 \mathrm{bp}$ upstream of the start of asr 0680 . This $92 \mathrm{bp}$ region corresponded to the region just upstream of asr0680 in Nostoc sp. strain PCC 7120 and Nodularia spumigena CCY 9414. These data supported the hypothesis that the location of asr0682 in Cylindrospermum sp. strain PCC 7417 is the result of a translocation. In addition, Nodularia spumigena CCY 9414 had a $1.5 \mathrm{~kb}$ segment of DNA that was also similar (85\%) to the all0684 and alr0686 of Nostoc sp. strain PCC 7120 (Fig. 2).

BLAST searches of the hupL element sequences revealed the expected similarities to the other hupL elements, but the remainder of each element had little similarity to any other sequence in the database.

\section{Phylogenetic analysis of xisA and xisC}

The xis $A$ aligned data matrix was 1302 characters in length (see Supplementary Fig. S1 in IJSEM Online) and ML analyses using the $\operatorname{TrN}+\mathrm{G} \quad(-\ln L=3772.39389)$ and GTR + G models $(-\ln L=3747.13269)$ produced two trees each, all four of which had similar topologies. Analysis of xisA produced results that were consistent with other cyanobacterial phylogenies (Henson et al., 2004). A strict consensus of the two trees derived from the $\mathrm{TrN}+\mathrm{G}$ method is presented in Fig. 3. This tree consists of two major clades, with Cylindrospermum sp. strains PCC 7417 and PCC 7604 at the base of the tree. The first clade is composed of all of the Anabaena and most of the Nostoc strains, interspersed within the clade. The remaining Nostoc strains are placed in a clade along with the Scytonema and Nodularia strains.

The xisC aligned data matrix was 1551 characters (see Supplementary Fig. S2) in length, and ML analyses using the $\mathrm{HKY}+\mathrm{G}(-\operatorname{lnL}=2919.18578)$ and $\mathrm{GTR}+\mathrm{G}$ models $(-\operatorname{lnL}=2896.23888)$ resulted in 61 and 26 trees, respectively, all of which had similar topologies. A strict consensus of the 61 trees generated by the HKY + G method is presented in Fig. 4. Within this tree, the Nostoc and Anabaena strains occurred in an unresolved polytomy at the base of the tree, sister to a clade containing the Cylindrospermum and Nodularia strains. Although analysis of $x i s C$ lacked resolution, the relationships among the strains were congruent with other published cyanobacterial phylogenies (Henson et al., 2004).
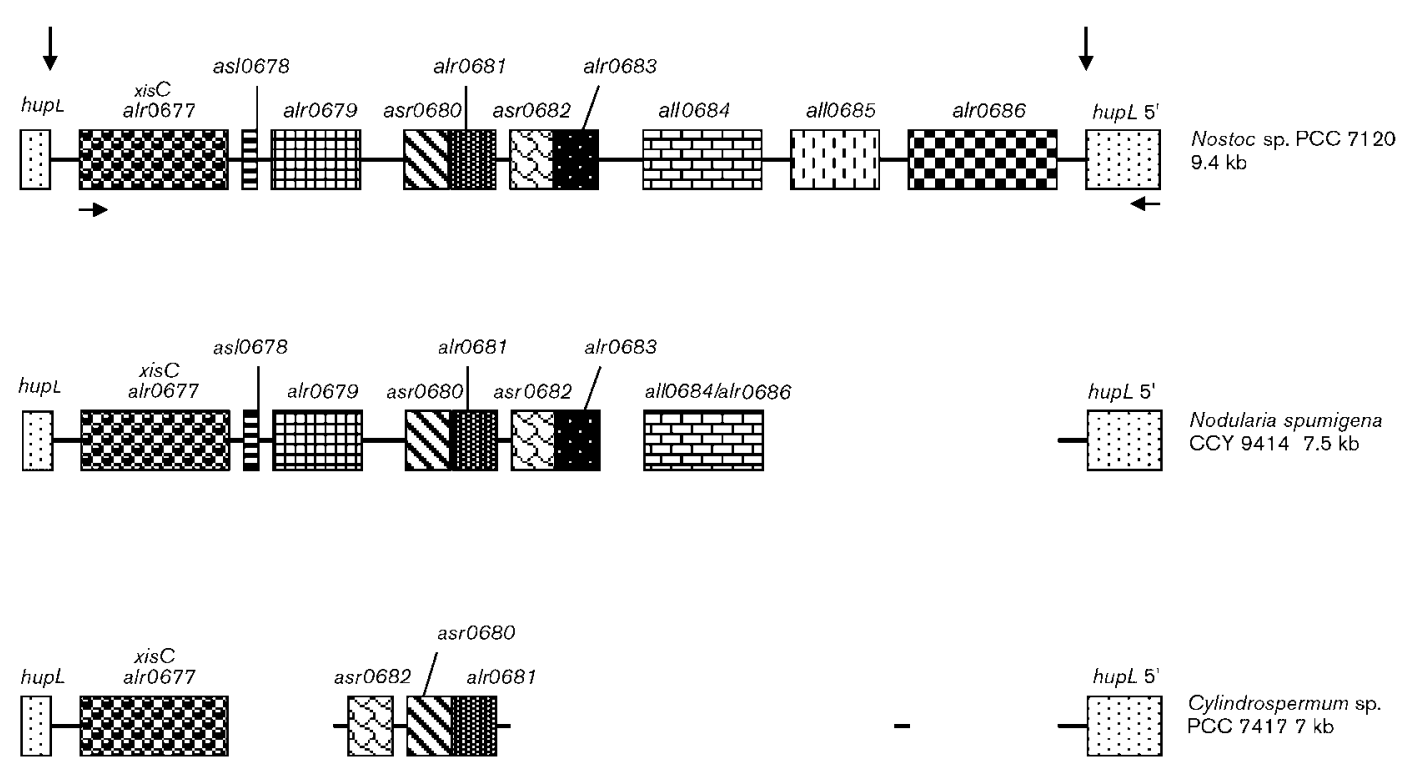

Fig. 2. A diagram showing the similarity between the hupL elements of Nostoc sp. strain PCC 7120, Nodularia spumigena CCY 9414 and Cylindrospermum sp. strain PCC 7417. All genes are labelled and represented by boxes that have different graphical patterns. The direct repeats are represented by vertical arrows and the horizontal arrows indicate the direction of transcription for hupL and xisC. Regions of the Nodularia spumigena CCY 9414 and Cylindrospermum sp. strain PCC 7417 elements that are similar to Nostoc sp. strain PCC 7120 are represented by boxes with identical graphical patterns and intergenic regions are represented by horizontal bars. 


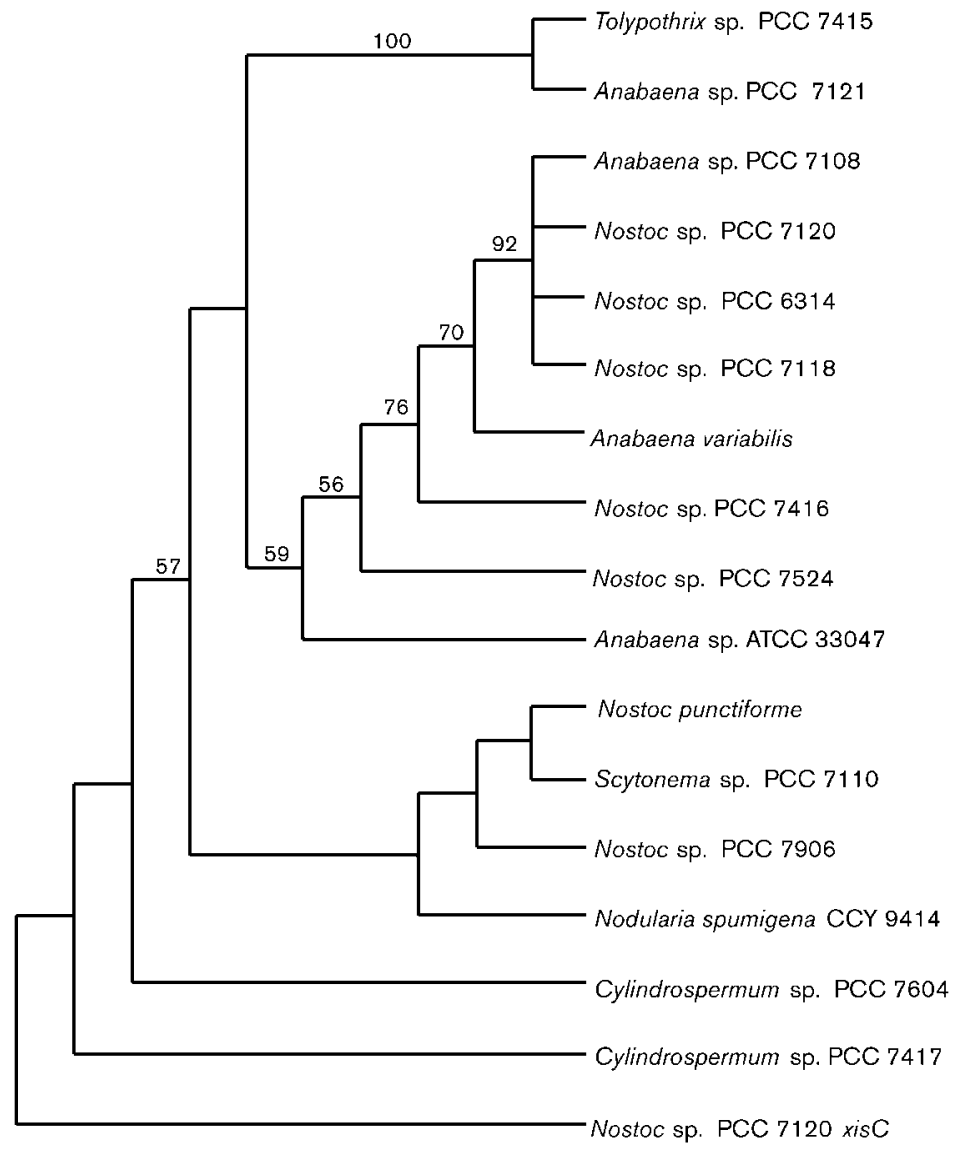

Fig. 3. A phylogenetic tree of $x i s A$ sequences created using $\mathrm{ML}$ analysis. Bootstrap values are listed above the branches.

\section{DISCUSSION}

Heterocyst differentiation in cyanobacteria is initiated by nitrogen deprivation. The transcription factor NtcA can gauge levels of environmental nitrogen levels (Muro-Pastor et al., 2001; Tanigawa et al., 2002; Herrero et al., 2004) and is believed to be the key regulator in initiating heterocyst differentiation and nitrogen fixation (Chastain et al., 1990; Jiang et al., 2000; Ramasubramanian et al., 1994, 1996; Herrero et al., 2004). Factors that induce the expression of $x i s C$ are unknown, however, the $x i s A$ promoter has binding sites for NtcA and FurA, suggesting that these transcription factors are involved in expression of xisA (Jiang et al., 2000; Ramasubramanian et al., 1994, 1996; Herrero et al., 2004; López-Gomollón et al., 2007). Understanding the evolutionary origin of the elements and their genes that encode self-excision may also provide insight into the origin and evolution of heterocyst formation.

There is considerable variation in size and composition within the representative nifD and $h u p L$ elements. The nifD element ranges from 4 to $24 \mathrm{~kb}$ and the hupL element ranges from 7 to $9.4 \mathrm{~kb}$. Neither element has much sequence similarity with other published sequences. However, there are conserved regions within each representative nifD and hupL element. Both element types have conserved $5^{\prime}$ and $3^{\prime}$ flanking regions, each element type encodes its own recombinase and, with the exception of Nodularia spumigena CCY 9414, each element type has a conserved central region. Phylogenetic analysis of $x i s A$ and $x i s C$ sequences resulted in phylogenies that were congruent with published heterocystous cyanobacterial phylogenies (Henson et al., 2004). This suggests that subsequent to the elements' initial insertion into the cyanobacterial lineage, they have undergone vertical descent and have not been transferred laterally within the lineage.

The evolutionary origin of the nifD and hupL elements remains unclear. The data suggest that they are the remnants of viruses or bacteriophages. This is supported by several lines of evidence. XisA and XisC belong to the tyrosine family of recombinases, which are mostly found in viruses (Nunes-Düby et al., 1998; Carrasco et al., 2005). In addition, the XisA and $\mathrm{XisC}$ sequences are more similar to each other than any other published sequence and both have the tyrosine to histidine mutation in the active site, suggesting that XisA and $\mathrm{XisC}$, as well as the nifD and hupL elements, share a common evolutionary origin.

Several structural aspects of the nifD and hupL elements suggest that they have a viral ancestry and may be bacteriophages that have lost the ability to self-replicate. The location of the XisA and XisC recombinases near the $5^{\prime}$ end of the nifD and hupL elements and the fact that the 


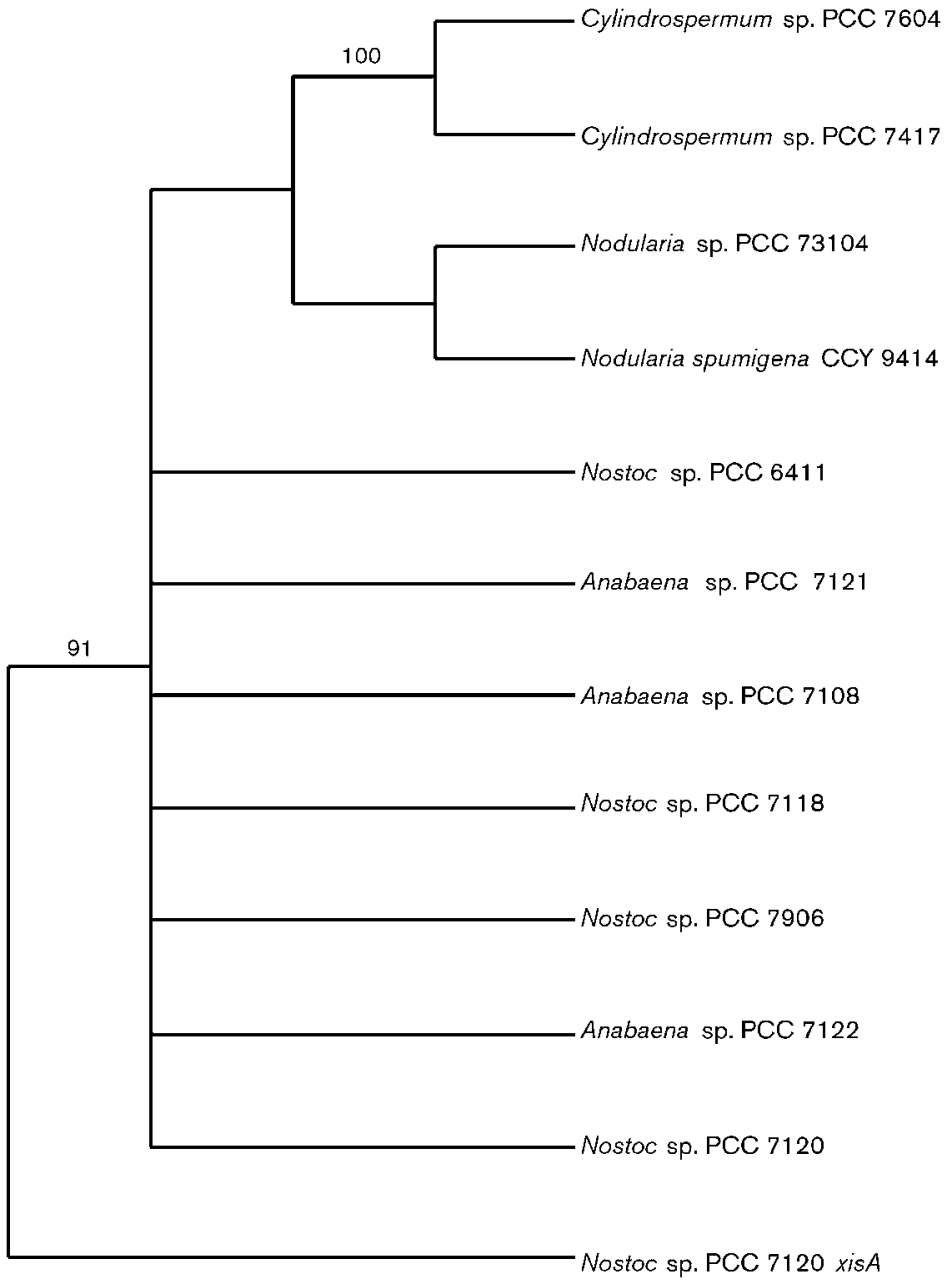

Fig. 4. A phylogenetic tree of $x i s C$ sequences created using $\mathrm{ML}$ analysis. Bootstrap values are listed above the branches.

elements are inserted in exactly the same location within the nifD and hupL genes in all examined strains suggest a viral origin (Casjens, 2003). However the only segment of any sequenced nifD or hupL element (other than $x i s A$ and $x i s C$ ) that is similar to a virus is the 125 bp region of the Anabaena sp. strain ATCC 33047 nifD element, which is similar to the Rabbit fibroma virus and Rat cytomegalovirus (Henson et al., 2005). This could suggest that the nifD or hupL elements are not of viral origin; however, these elements may represent defective prophages that are in a state of mutational decay (Casjens, 2003; Canchaya et al., 2003). Defective prophages in a state of mutational decay are characterized by having increased mutational rates in select regions as well as having large-scale deletions (Casjens, 2003; Canchaya et al., 2003). In defective prophages, it is possible for certain genes to be retained through selection, while the remainder of the prophage accumulates mutations. This could explain why there is so much structural and compositional variation within the nifD and hupL elements, yet certain regions remain conserved. It is also possible for a highly mutated prophage to have diverged so much that no characteristic genes from the virus, other than the recombinase, remain (Casjens, 2003).
If the nifD and hupL elements are defective prophages, they exist as part of the flexible gene pool in cyanobacteria. The flexible gene pool is best described as the DNA within a bacterial genome that arose through horizontal transfer (Hacker \& Carniel, 2001). The flexible gene pool consists of genetic entities such as genomic islands, prophages, integrons, transposons, genomic islets and plasmids (Hacker \& Carniel, 2001). Genetic information within the flexible gene pool may provide an ecological or pathogenic advantage; however it could also provide no selective advantage and exists as selfish DNA (Lilley et al., 2000).

Although the nifD and hupL elements may be descended from a common ancestor, their insertion into the nifD and hupL genes probably occurred as two separate events. If the nifD and hupL elements resulted from a single insertion event (i.e. a single origin) into the cyanobacteria and subsequently underwent duplication and divergence, we would expect more sequence conservation between the two than is currently present. Other than $x i s A$ and $x i s C$, which have similar nucleotide and amino acid sequences, the nifD elements share no sequence similarity to the hupL elements. In addition to their independent insertions, the nifD and 
hupL elements have probably been under different selection pressures, which might explain the haphazard occurrence of the elements within the cyanobacteria. Initially both elements were thought to only be in Subsection IV, which is the case for the nifD element. However, the hupL element (or at least a portion of $x i s C$ ) has been found within a Subsection V Fischerella strain and a Subsection III Leptolyngbya strain (Tamagnini et al., 2000), suggesting that the hupL element may have originated within the lineage prior to the diversification of the filamentous cyanobacteria (Subsections III, IV and V).

Individual regions within each element type may be under different selection pressures and have experienced differential rates of evolution. This would explain the extensive structural and compositional variation within the nifD and hupL elements that is coupled with the presence of conserved regions. If only specific regions of the nifD and $h u p L$ elements are under selective pressure, then the remaining portions of each element would be free to accumulate mutations (e.g. insertions, deletions, and translocations) without inhibiting excision or being detrimental to the organism.

For DNA elements to be maintained in the cyanobacterial genomes, in theory there should be some sort of selective pressure to retain them. Although the Nostoc punctiforme and Cylindrospermum sp. strain PCC 7417 nifD elements contain tRNA-Asn and ctiA genes, the only known functional products encoded within the nifD and hupL elements are xisA and xisC. Thus, the only selective pressure on the nifD and hupL elements may be for their successful excision. A mutation inhibiting excision of either element would drastically affect nitrogen fixation and would be detrimental to the organism.

The variation in size, structure, and composition of nifD and hupL elements indicates a complex pattern of insertions, deletions, translocation and sequence divergence over the course of evolution. This complex evolutionary pattern suggests that, aside from the conserved regions, these elements are a repository of genetic information where segments of DNA can undergo mutations with little to no effect on the organism or element excision. The nifD and $h u p L$ elements represent an interesting aspect of the regulation of nitrogen fixation, heterocyst differentiation and the molecular evolution of genome structure within the heterocystous cyanobacteria. In addition, they may represent an excellent system for the study of defective prophages that are in advanced stages of mutational decay.

\section{REFERENCES}

Adams, D. G. \& Duggan, P. S. (1999). Heterocyst and akinete differentiation in cyanobacteria. New Phytol 144, 3-33.

Alvey, R. M., Karty, J. A., Roos, E., Reilly, J. P. \& Kehoe, D. M. (2003). Lesions in phycoerythrin chromophore biosynthesis in Fremyella diplosiphon reveal coordinated light regulation of apoprotein and pigment biosynthetic enzyme gene expression. Plant Cell 15, 2448-2463.
Böhme, H. (1998). Regulation of nitrogen fixation in heterocystforming cyanobacteria. Trends Plant Sci 3, 346-351.

Bremer, K. (1994). Branch support and tree stability. Cladistics 10, 295-304.

Brusca, J. S., Hale, M. A., Carrasco, C. D. \& Golden, J. W. (1989). Excision of an 11-kilobase-pair DNA element from within the nifD gene in Anabaena variabilis heterocysts. J Bacteriol 171, 4138-4145.

Brusca, J. S., Chastain, C. J. \& Golden, J. W. (1990). Expression of the Anabaena sp. strain PCC 7120 xisA gene from a heterologous promoter results in excision of the nifD element. J Bacteriol 172, 3925-3931.

Canchaya, C., Proux, C., Fournous, G., Bruttin, A. \& Brüssow, H. (2003). Prophage genomics. Microbiol Mol Biol Rev 67, 238-276.

Carrasco, C. D. \& Golden, J. W. (1995). Two heterocyst-specific DNA rearrangements of nif operons in Anabaena cylindrica and Nostoc sp. strain Mac. Microbiology 141, 2479-2487.

Carrasco, C. D., Ramaswamy, K. S., Ramasubramanian, T. S. \& Golden, J. W. (1994). Anabaena $x i s F$ gene encodes a developmentally regulated site-specific recombinase. Genes Dev 8, 74-83.

Carrasco, C. D., Buettner, J. A. \& Golden, J. W. (1995). Programmed DNA rearrangement of a cyanobacterial hupL gene in heterocysts. Proc Natl Acad Sci U S A 92, 791-795.

Carrasco, C. D., Holliday, S. D., Hansel, A., Lindblad, P. \& Golden, J. W. (2005). Heterocyst-specific excision of the Anabaena sp. strain PCC 7120 hupL element requires xisC. J Bacteriol 187, 6031-6038.

Casjens, S. (2003). Prophages and bacterial genomics: what have we learned so far? Mol Microbiol 49, 277-300.

Chastain, C. J., Brusca, J. S., Ramasubramanian, T. S., Wei, T. F. \& Golden, J. W. (1990). A sequence-specific DNA-binding factor (VF1) from Anabaena sp. strain PCC 7120 vegetative cells binds to three adjacent sites in the xisA upstream region. J Bacteriol 172, 50045051.

Felsenstein, J. (1985). Confidence limits on phylogenies: an approach using the bootstrap. Evol 39, 783-791.

Golden, J. W., Robinson, S. J. \& Haselkorn, R. (1985). Rearrangement of nitrogen fixation genes during heterocyst differentiation in the cyanobacterium Anabaena. Nature 314, 419-423.

Golden, J. W., Mulligan, M. E. \& Haselkorn, R. (1987). Different recombination site specificity of two developmentally regulated genome rearrangements. Nature 327, 526-529.

Golden, J. W., Carrasco, C. D., Mulligan, M. E., Schneider, G. J. \& Haselkorn, R. (1988). Deletion of a 55-kilobase-pair DNA element from the chromosome during heterocyst differentiation of Anabaena sp. strain PCC 7120. J Bacteriol 170, 5034-5041.

Golden, J. W., Whorff, L. L. \& Wiest, D. R. (1991). Independent regulation of nifHDK operon transcription and DNA rearrangement during heterocyst differentiation in the cyanobacterium Anabaena sp. strain PCC 7120. J Bacteriol 173, 7098-7105.

Hacker, J. \& Carniel, E. (2001). Ecological fitness, genomic islands and bacterial pathogenicity. A Darwinian view of the evolution of microbes. EMBO Rep 2, 376-381.

Hall, B. G. (2004). Codon Align. Macintosh, Windows, Unix. Distributed by the author. Bellingham, WA.

Happe, T., Schütz, K. \& Böhme, H. (2000). Transcriptional and mutational analysis of the uptake hydrogenase of the filamentous cyanobacterium Anabaena variabilis ATCC 29413. J Bacteriol 182, 1624-1631.

Haraldsen, J. D. \& Sonenshein, A. L. (2003). Efficient sporulation in Clostridium difficile requires disruption of the sigmaK gene. $\mathrm{Mol}$ Microbiol 48, 811-821. 
Haselkorn, R. (1992). Developmentally regulated gene rearrangements in prokaryotes. Annu Rev Genet 26, 113-130.

Henson, B. J., Watson, L. E. \& Barnum, S. R. (2002). Molecular differentiation of the heterocystous cyanobacteria, Nostoc and Anabaena, based on complete NifD sequences. Curr Microbiol 45, 161-164.

Henson, B. J., Hesselbrock, S. M., Watson, L. E. \& Barnum, S. R. (2004). Molecular phylogeny of the heterocystous cyanobacteria (subsections IV and V) based on nifD. Int J Syst Evol Microbiol 54, 493-497.

Henson, B. J., Watson, L. E. \& Barnum, S. R. (2005). Characterization of a $4 \mathrm{~kb}$ variant of the nifD element in Anabaena sp. strain ATCC 33047. Curr Microbiol 50, 129-132.

Herrero, A., Muro-Pastor, A. M., Valladares, A. \& Flores, E. (2004), Cellular differentiation and the NtcA transcription factor in filamentous cyanobacteria. FEMS Microbiol Rev 28, 469-487.

Huelsenbeck, J. P., Hillis, D. M. \& Jones, R. (1995). Parametric bootstrapping in molecular phylogenetics: applications and performance. In Molecular Zoology: Advances, Strategies and Protocols, pp. 19-46. Edited by J. S. Ferraris \& S. R. Palumbi. NY: Wiley-Liss.

Jiang, F. Y., Wisén, S., Widersten, M., Bergman, B. \& Mannervik, B. (2000). Examination of the transcription factor NtcA-binding motif by in vitro selection of DNA sequences from a random library. $J \mathrm{Mol}$ Biol 301, 783-793.

Kallas, T., Coursin, T. \& Rippka, R. (1985). Different organization of nif genes in nonheterocystous and heterocystous cyanobacteria. Plant Mol Biol 5, 321-329.

Kaneko, T., Nakamura, Y., Wolk, C. P., Kuritz, T., Sasamoto, S., Watanabe, A., Iriguchi, M., Ishikawa, A., Kawashima, K. \& other authors (2001). Complete genomic sequence of the filamentous nitrogen-fixing cyanobacterium Anabaena sp. strain PCC 7120. DNA Res 8, 205-213, 227-253.

Kunkel, B., Losick, R. \& Stragier, P. (1990). The Bacillus subtilis gene for the development transcription factor sigma $\mathrm{K}$ is generated by excision of a dispensable DNA element containing a sporulation recombinase gene. Genes Dev 4, 525-535.

Lammers, P. J., Golden, J. W. \& Haselkorn, R. (1986). Identification and sequence of a gene required for a developmentally regulated DNA excision in anabaena. Cell 44, 905-911.

Lammers, P. J., McLaughlin, S., Papin, S., Trujillo-Provencio, C. \& Ryncarz, A. J., II (1990). Developmental rearrangement of cyanobacterial nif genes: nucleotide sequence, open reading frames, and cytochrome P-450 homology of the Anabaena sp. strain PCC 7120 nifD element. J Bacteriol 172, 6981-6990.

Lilley, A., Young, P. \& Bailey, M. (2000). Bacterial population genetics: do plasmids maintain bacterial diversity and adaptation? In The Horizontal Gene Pool, pp. 287-300. Edited by C. M. Thomas. Amsterdam: Harwood Academic Publishers.

López-Gomollón, S., Hernández, J. A., Pellicer, S., Angarica, V. E., Peleato, M. L. \& Fillat, M. F. (2007). Cross-talk between iron and nitrogen regulatory networks in Anabaena (Nostoc) sp. PCC 7120 identification of overlapping genes in FurA and NtcA regulons. J Mol Biol 374, 267-281.

Lynn, M. E., Bantle, J. A. \& Ownby, J. D. (1986). Estimation of gene expression in heterocysts of Anabaena variabilis by using DNA-RNA hybridization. J Bacteriol 167, 940-946.

Maruyama, K., Sato, N. \& Ohta, N. (1999). Conservation of structure and cold-regulation of RNA-binding proteins in cyanobacteria: probable convergent evolution with eukaryotic glycine-rich RNAbinding proteins. Nucleic Acids Res 27, 2029-2036.

Mazur, B. J. \& Chui, C. F. (1982). Sequence of the gene coding for the beta-subunit of dinitrogenase from the blue-green alga Anabaena. Proc Natl Acad Sci U S A 79, 6782-6786.
Meeks, J. C., Joseph, C. M. \& Haselkorn, R. (1988). Organization of the nif genes in cyanobacteria in symbiotic association with Azolla and Anthoceros. Arch Microbiol 150, 61-71.

Meeks, J. C., Campbell, E. L. \& Bisen, P. S. (1994). Elements interrupting nitrogen-fixation genes in cyanobacteria - presence and absence of a nifD in clones of Nostoc sp. strain Mac. Microbiology 140, 3225-3232.

Meeks, J. C., Elhai, J., Thiel, T., Potts, M., Larimer, F., Lamerdin, J., Predki, P. \& Atlas, R. (2001). An overview of the genome of Nostoc punctiforme, a multicellular, symbiotic cyanobacterium. Photosynth Res 70, 85-106.

Mulligan, M. E., Buikema, W. J. \& Haselkorn, R. (1988). Bacterial-type ferredoxin genes in the nitrogen fixation regions of the cyanobacterium Anabaena sp. strain PCC 7120 and Rhizobium meliloti. J Bacteriol 170, 4406-4410.

Muro-Pastor, M. I., Reyes, J. C. \& Florencio, F. J. (2001). Cyanobacteria perceive nitrogen status by sensing intracellular 2oxoglutarate levels. J Biol Chem 276, 38320-38328.

Nunes-Düby, S. E., Kwon, H. J., Tirumalai, R. S., Ellenberger, T. \& Landy, A. (1998). Similarities and differences among 105 members of the Int family of site-specific recombinases. Nucleic Acids Res 26, 391406.

Okada, K., Kamiya, Y., Zhu, X. F., Suzuki, K., Tanaka, K., Nakagawa, T., Matsuda, H. \& Kawamukai, M. (1997). Cloning of the $s d s$ A gene encoding solanesyl diphosphate synthase from Rhodobacter capsulatus and its functional expression in Escherichia coli and Saccharomyces cerevisiae. J Bacteriol 179, 5992-5998.

Oxelfelt, F., Tamagnini, P., Salema, R. \& Lindblad, P. (1995). Hydrogen uptake in Nostoc strain PCC-73102 - effects of nickel, hydrogen, carbon, and nitrogen. Plant Physiol Biochem 33, 617-623.

Posada, D. \& Crandall, K. A. (1998). MODELTEST: testing the model of DNA substitution. Bioinformatics 14, 817-818.

Prasanna, R. \& Kaushik, B. D. (1995). Nitrogen fixation and nif gene organization in branched heterocystous cyanobacteria: variation in the presence of xisA. Folia Microbiol (Praha) 40, 176-180.

Ramasubramanian, T. S., Wei, T. F. \& Golden, J. W. (1994). Two Anabaena sp. strain PCC 7120 DNA-binding factors interact with vegetative cell- and heterocyst-specific genes. J Bacteriol 176, 12141223.

Ramasubramanian, T. S., Wei, T. F., Oldham, A. K. \& Golden, J. W. (1996). Transcription of the Anabaena sp. strain PCC 7120 ntcA gene: multiple transcripts and NtcA binding. J Bacteriol 178, 922-926.

Ramaswamy, K. S., Carrasco, C. D., Fatma, T. \& Golden, J. W. (1997). Cell-type specificity of the Anabaena $f d x \mathrm{~N}$-element rearrangement requires xis $\mathrm{H}$ and xisI. Mol Microbiol 23, 1241-1249.

Rice, D., Mazur, B. J. \& Haselkorn, R. (1982). Isolation and physical mapping of nitrogen fixation genes from the cyanobacterium Anabaena 7120. J Biol Chem 257, 13157-13163.

Saville, B., Straus, N. \& Coleman, J. R. (1987). Contiguous organization of nitrogenase genes in a heterocystous cyanobacterium. Plant Physiol 85, 26-29.

Sazuka, T. (2003). Proteomic analysis of the cyanobacterium Anabaena sp. strain PCC 7120 with two-dimensional gel electrophoresis and amino-terminal sequencing. Photosynth Res 78, 279-291.

Smith, M. C. M. \& Thorpe, H. M. (2002). Diversity in the serine recombinases. Mol Microbiol 44, 299-307.

Tamagnini, P., Costa, J. L., Almeida, L., Oliveira, M. J., Salema, R. \& Lindblad, P. (2000). Diversity of cyanobacterial hydrogenases, a molecular approach. Curr Microbiol 40, 356-361.

Tanigawa, R., Shirokane, M., Maeda Si, S., Omata, T., Tanaka, K. \& Takahashi, H. (2002). Transcriptional activation of NtcA-dependent 
promoters of Synechococcus sp. PCC 7942 by 2-oxoglutarate in vitro. Proc Natl Acad Sci U S A 99, 4251-4255.

Thompson, J. D., Higgins, D. G. \& Gibson, T. J. (1994). Clustal W: improving the sensitivity of progressive multiple sequence alignment through sequence weighting, position-specific gap penalties and weight matrix choice. Nucleic Acids Res 22, 4673-4680.
Voziyanov, Y., Pathania, S. \& Jayaram, M. (1999). A general model for site-specific recombination by the integrase family recombinases. Nucleic Acids Res 27, 930-941.

Wolk, P. C., Anneliese, E. \& Elhai, J. (1994). Heterocyst metabolism and development. In The Molecular Biology of Cyanobacteria, pp. 769-823. Edited by D. A. Bryant. The Netherlands: Kluwer Academic Publishers. 